\title{
A REMARK ON HOMOGENEOUS LINEAR EQUATIONS WITH VARIABLE COEFFICIENTS
}

\author{
By YoshikazU HiRAsAwa
}

1. We use the same notations as in previous papers [1], [2]. Let $I$ be a closed interval $[\alpha, \beta]=\{t \mid \alpha \leqq t \leqq \beta, t \in \mathbf{R}\}$. We denote by $C^{\mu}(I, \mathbf{C})$ the totality of complex-valued functions defined and of class $C^{\mu}$ on $I(\mu=0,1, \cdots, \infty)$ and hereafter we fix some $\mu$.

For the sake of brevity, we denote $C^{\mu}(I, \mathbf{C})$ by $K(I)$ and $K(I)^{n}$ by $M(I)$ :

$$
M(I)=\left\{\boldsymbol{f}(t)=\operatorname{col}\left(f_{1}(t), f_{2}(t), \cdots, f_{n}(t) \mid f_{j}(t) \in K(I), \quad j=1,2, \cdots, n\right\} .\right.
$$

Now let $B(t)$ be a square matrix of degree $n$ whose components all belong to $K(I)$ :

$$
B(t)=\left(\begin{array}{cccc}
b_{11}(t) & b_{12}(t) & \cdots & b_{1 n}(t) \\
b_{21}(t) & b_{22}(t) & \cdots & b_{2 n}(t) \\
\vdots & \vdots & & \vdots \\
b_{n 1}(t) & b_{n 2}(t) & \cdots & b_{n n}(t)
\end{array}\right),
$$

and let us assume, throughout this paper, that for a positive integer $s: 1 \leqq s \leqq n-1$, a condition

$$
\operatorname{rank} B(t)=n-s
$$

is satisfied on $I$.

We consider a homogeneous linear equation

$$
B(t) \boldsymbol{f}(t)=\boldsymbol{o} \quad \text { on } \quad I ; \quad \boldsymbol{f}(t) \in M(I),
$$

and we denote the totality of solutions of equation (3) by $W(I)$ :

$$
W(I)=\{\boldsymbol{f}(t) \in M(I) \mid B(t) \boldsymbol{f}(t)=\boldsymbol{o} \text { on } I\} .
$$

Then, we know that there exist $s$ vectors

$$
\boldsymbol{p}_{g}(t)=\operatorname{col}\left(p_{1 g}(t), p_{2 g}(t), \cdots, p_{n g}(t)\right) \quad(g=1,2, \cdots, s)
$$

belonging to $W(I)$, such that

$$
\operatorname{rank}\left(\boldsymbol{p}_{1}(t), \boldsymbol{p}_{2}(t), \cdots, \boldsymbol{p}_{\boldsymbol{s}}(t)\right)=s
$$

Received December 12, 1985 
for any $t \in I$.

For a proof of this fact, see, for example, the proof of Theorem in the previous paper [1].

The purpose of this paper is to clarify the relation between the vectors $\boldsymbol{p}_{g}(t)(g=1,2, \cdots, s)$ given above and any vector

$$
\boldsymbol{u}(t)=\operatorname{col}\left(u_{1}(t), u_{2}(t), \cdots, u_{n}(t)\right)
$$

belonging to $W(I)$. A result for this theme will be stated as Theorem in No. 3 .

Let $Q(t)$ be an $n \times s$ matrix whose components all belong to $K(I)$ :

$$
Q(t)=\left(\begin{array}{cccc}
q_{11}(t) & q_{12}(t) & \cdots & q_{1 s}(t) \\
q_{21}(t) & q_{22}(t) & \cdots & q_{2 s}(t) \\
\vdots & \vdots & & \vdots \\
q_{n 1}(t) & q_{n 2}(t) & \cdots & q_{n s}(t)
\end{array}\right),
$$

where $s$ is an integer such that $1 \leqq s \leqq n-1$. We denote, in general, a minor of degree $s$ of the matrix $Q(t)$, by

$$
\begin{array}{r}
Q\left(\begin{array}{cccc}
i_{1} & i_{2} & \cdots & i_{s} \\
1 & 2 & \cdots & s
\end{array}\right)= \\
\left|\begin{array}{cccc}
q_{\imath_{1} 1}(t) & q_{\imath_{1}}(t) & \cdots & q_{\imath_{1} s}(t) \\
q_{2_{2} 1^{1}}(t) & q_{2_{2}}(t) & \cdots & q_{\imath_{2} s}(t) \\
\vdots & \vdots & & \vdots \\
q_{\imath_{s} 1}(t) & q_{\imath_{s}}(t) & \cdots & q_{\imath_{s} s}(t)
\end{array}\right| \\
\left(1 \leqq i_{1}<i_{2}<\cdots<i_{s} \leqq n\right) .
\end{array}
$$

2. We give the following two lemmas:

LEMMA 1. Let $Q(t)$ be an $n \times s$ matrix whose components all belong to $K(I)$, where $s$ is an integer such that $1 \leqq s \leqq n-1$, and suppose that a condition

$$
\operatorname{rank} Q(t)=s
$$

is satisfied on $I$.

Let $I_{0}$ be a subinterval of $I$ and let

$$
\boldsymbol{x}(t)=\operatorname{col}\left(x_{1}(t), x_{2}(t), \cdots, x_{s}(t)\right)
$$

be an s-dimensional vector such that $x_{g}(t) \in K\left(I_{0}\right)(g=1,2, \cdots, s)$ and

$$
Q(t) \boldsymbol{x}(t)=\boldsymbol{o} \text { on } I_{0} .
$$

Then we have $\boldsymbol{x}(t) \equiv \boldsymbol{o}$ on $I_{0}$.

Proof. For any $t_{0} \in I_{0}$, we can choose, by assumption, a minor $Q\left(\begin{array}{cccc}l_{1} & i_{2} & \cdots & i_{s} \\ 1 & 2 & \cdots & s\end{array}\right)$ of degree $s$ of $Q(t)$, not vanishing at $t_{0}$.

Putting

$$
\tilde{\boldsymbol{q}}_{g}(t)=\operatorname{col}\left(q_{\imath_{1} g}(t), q_{\imath_{2} g}(t), \cdots, q_{\imath_{s} g}(t)\right) \quad(g=1,2, \cdots, s),
$$


we have

$$
\operatorname{det}\left(\tilde{\boldsymbol{q}}_{1}\left(t_{0}\right), \tilde{\boldsymbol{q}}_{2}\left(t_{0}\right), \cdots, \tilde{\boldsymbol{q}}_{s}\left(t_{0}\right)\right) \neq 0
$$

and in virtue of the relation (6), we get

$$
\sum_{g=1}^{s} x_{g}\left(t_{0}\right) \tilde{\boldsymbol{q}}_{\boldsymbol{g}}\left(t_{0}\right)=\boldsymbol{o} .
$$

Hence the condition (7) implies $\boldsymbol{x}\left(t_{0}\right)=\boldsymbol{o}$ and since $t_{0}$ is any point in $I_{0}$, we see $\boldsymbol{x}(t) \equiv \boldsymbol{o}$ on $I_{0}$.

LEMMA 2. Let $\boldsymbol{p}_{g}(t)(g=1,2, \cdots, s)$ be $s$ vectors belonging to $W(I)$ and satisfying the condition (4) on $I$, and let $\boldsymbol{u}(t)$ be any vector belonging to $W(I)$.

Then we have

$$
\operatorname{rank}\left(\boldsymbol{p}_{1}(t), \cdots, \boldsymbol{p}_{s}(t), \boldsymbol{u}(t)\right)=s \quad \text { on } \quad I .
$$

Proof. We put $P(t)=\left(\boldsymbol{p}_{1}(t), \boldsymbol{p}_{2}(t), \cdots, \boldsymbol{p}_{s}(t)\right)$ and then we have always

$$
s \leqq \operatorname{rank}(P(t), \boldsymbol{u}(t)) \leqq s+1 \quad \text { on } \quad I .
$$

If there were a point $t_{0} \in I$ such that

$$
\operatorname{rank}\left(P\left(t_{0}\right), \boldsymbol{u}\left(t_{0}\right)\right)=s+1,
$$

then, since

we should obtain

$$
B\left(t_{0}\right)\left(P\left(t_{0}\right), \boldsymbol{u}\left(t_{0}\right)\right)=O,
$$

$$
\operatorname{rank} B\left(t_{0}\right) \leqq n-(s+1) \text {; }
$$

this, however, contradicts the condition (2). Therefore we have always the equality (8) on $I$.

3. We shall prove the following theorem:

THEOREM. Let

$$
\boldsymbol{p}_{g}(t)=\operatorname{col}\left(p_{1 g}(t), p_{2 g}(t), \cdots, p_{n g}(t)\right) \quad(g=1,2, \cdots, s)
$$

be s vectors belonging to $W(I)$ and satisfying the condition (4) on $I$, and let

$$
\boldsymbol{u}(t)=\operatorname{col}\left(u_{1}(t), u_{2}(t), \cdots, u_{n}(t)\right)
$$

be any vector belonging to $W(I)$.

Then we can represent $\boldsymbol{u}(t)$ uniquely as a linear combination of the vectors $\boldsymbol{p}_{\boldsymbol{g}}(t)(g=1,2, \cdots, s)$ with coefficients $\zeta_{\boldsymbol{g}}(t)(g=1,2, \cdots, s)$ belonging to $K(I)$ :

$$
\boldsymbol{u}(t)=\sum_{g=1}^{s} \zeta_{g}(t) \boldsymbol{p}_{g}(t)
$$

on $I$. 
Proof. We put $P(t)=\left(\boldsymbol{p}_{1}(t), \boldsymbol{p}_{2}(t), \cdots, \boldsymbol{p}_{s}(t)\right)$.

There exists, in virtue of the condition (4), a set $\left\{I_{\kappa}\right\}_{\kappa=1}^{\kappa_{0}}$ of subintervals of $I$, possessing the following properties:

(i) $I=\bigcup_{\kappa=1}^{\kappa_{0}} I_{\kappa}$;

(ii) $I_{1}=\left[\alpha_{1}, \beta_{1}\right), I_{\kappa_{0}}=\left(\alpha_{\kappa_{0}}, \beta_{\kappa_{0}}\right], \alpha_{1}=\alpha, \beta_{\kappa_{0}}=\beta$, $I_{\kappa}=\left(\alpha_{\kappa}, \beta_{\kappa}\right)\left(\kappa=2,3, \cdots, \kappa_{0}-1\right)$;

(iii) $I_{\kappa} \cap I_{\kappa+1} \neq \varnothing\left(\kappa=1,2, \cdots, \kappa_{0}-1\right), I_{\kappa} \cap I_{\kappa^{\prime}}=\varnothing\left(\kappa+1<\kappa^{\prime}, \kappa=1,2, \cdots, \kappa_{0}-2\right)$, that is, $\alpha_{1}<\alpha_{2}<\beta_{1}<\cdots<\alpha_{\kappa}<\beta_{\kappa-1}<\alpha_{\kappa+1}<\beta_{\kappa}<\cdots<\beta_{\kappa_{0}-2}<\alpha_{\kappa_{0}}<\beta_{\kappa_{0}-1}<\beta_{\kappa_{0}}$ $\left(\kappa=2,3, \cdots, \kappa_{0}-1\right)$;

(iv) For each $I_{\kappa}$, there exists a minor of degree $s$ of $P(t)$, which does not vanish on $I_{\kappa}$.

We consider first the interval $I_{1}$ and choose a minor $P\left(\begin{array}{cccc}i_{1} & i_{2} & \cdots & i_{s} \\ 1 & 2 & \cdots & s\end{array}\right)$ of degree $s$ of $P(t)$ such that a condition

$$
P\left(\begin{array}{cccc}
i_{1} & i_{2} & \cdots & i_{s} \\
1 & 2 & \cdots & s
\end{array}\right) \neq 0
$$

is satisfied on $I_{1}$.

Let us define $(n-s)$-tuple $\left(i_{s+1}^{\prime}, i_{s+2}^{\prime}, \cdots, i_{n}^{\prime}\right)$ for $1 \leqq l_{1}<i_{2}<\cdots<i_{s} \leqq n$ in such a way that $1 \leqq i_{s+1}^{\prime}<i_{s+2}^{\prime}<\cdots<i_{n}^{\prime} \leqq n$ and $\left\{i_{1}, \cdots, i_{s}, i_{s+1}^{\prime}, \cdots, i_{n}^{\prime}\right\}=\{1,2, \cdots, n\}$ and put

$$
\begin{aligned}
& \tilde{\boldsymbol{p}}_{g}(t)=\operatorname{col}\left(p_{i_{1} g}(t), p_{i_{2} g}(t), \cdots, p_{i_{s} g}(t)\right) \quad(g=1,2, \cdots, s), \\
& \tilde{\boldsymbol{p}}_{g}^{\prime}(t)=\operatorname{col}\left(p_{i_{s+1}^{\prime} g}(t), p_{i_{s+2}^{\prime} g}(t), \cdots, p_{i^{\prime} g}(t)\right) \quad(g=1,2, \cdots, s), \\
& \tilde{\boldsymbol{u}}(t)=\operatorname{col}\left(u_{\imath_{1}}(t), u_{\imath_{2}}(t), \cdots, u_{\imath_{s}}(t)\right), \\
& \tilde{\boldsymbol{u}}^{\prime}(t)=\operatorname{col}\left(u_{i_{s+1}^{\prime}}(t), u_{i_{s+2}^{\prime}}(t), \cdots, u_{i_{n}^{\prime}}(t)\right) .
\end{aligned}
$$
know

Since, in virtue of the fact that the condition (10) is satisfied on $I_{1}$, we

$$
\operatorname{det}\left(\tilde{\boldsymbol{p}}_{1}(t), \tilde{\boldsymbol{p}}_{2}(t), \cdots, \tilde{\boldsymbol{p}}_{s}(t)\right) \neq 0 \quad \text { on } \quad I_{1},
$$

we can represent the vector $\tilde{\boldsymbol{u}}(t)$ as a linear combination of the vectors $\tilde{\boldsymbol{p}}_{g}(t)$ $(g=1,2, \cdots, s)$ with coefficients $\zeta_{g}(t)(g=1,2, \cdots, s)$ belonging to $K\left(I_{1}\right)$ :

$$
\tilde{\boldsymbol{u}}(t)=\sum_{g=1}^{s} \zeta_{g}(t) \tilde{\boldsymbol{p}}_{g}(t)
$$

on $I_{1}$.

Furthermore, we wish to show that the vector $\boldsymbol{u}(t)$ can be represented on $I_{1}$, as a linear combination (9) of the vectors $\boldsymbol{p}_{g}(t)(g=1,2, \cdots, s)$ with the same coefficients $\zeta_{g}(t)(g=1,2, \cdots, s)$ as in the representation (11). To this end, we have only to prove that a representation 


$$
\tilde{\boldsymbol{u}}^{\prime}(t)=\sum_{g=1}^{s} \zeta_{g}(t) \tilde{\boldsymbol{p}}_{g}^{\prime}(t)
$$

holds on $I_{1}$.

If there were a point $t_{0} \in I_{1}$ and an index $\rho(s+1 \leqq \rho \leqq n)$ such that

$$
u_{i^{\prime} \rho}\left(t_{0}\right) \neq \sum_{g=1}^{s} \zeta_{g}\left(t_{0}\right) p_{i^{\prime} \rho g}\left(t_{0}\right),
$$

then we should have

$$
\operatorname{rank}\left(P\left(t_{0}\right), \boldsymbol{u}\left(t_{0}\right)\right)=s+1 .
$$

This equality contradicts the result which was proved in Lemma 2 . Therefore we have the representation (12) on $I_{1}$.

Next we consider the second interval $I_{2}$ and then, in the same way as for the interval $I_{1}$, we get a representation

$$
\boldsymbol{u}(t)=\sum_{g=1}^{s} \theta_{g}(t) \boldsymbol{p}_{\boldsymbol{g}}(t)
$$

on $I_{2}$, where $\theta_{g}(t) \in K\left(I_{2}\right)(g=1,2, \cdots, s)$.

It follows from the representations (9) on $I_{1}$ and (13) on $I_{2}$, that

$$
\sum_{g=1}^{s}\left(\zeta_{g}(t)-\theta_{g}(t)\right) \boldsymbol{p}_{g}(t) \equiv \boldsymbol{o}
$$

on $I_{1} \cap I_{2}$, and hence by Lemma 1 , we obtain

$$
\zeta_{g}(t) \equiv \theta_{g}(t) \quad(g=1,2, \cdots, s) \quad \text { on } \quad I_{1} \cap I_{2} .
$$

Therefore, by defining functions $\zeta_{g}(t) \in K\left(I_{1} \cup I_{2}\right)(g=1,2, \cdots, s)$ as follows :

$$
\zeta_{g}(t)=\left\{\begin{array}{lll}
\zeta_{g}(t) & \text { on } & I_{1}, \\
\theta_{g}(t) & \text { on } & I_{2},
\end{array}\right.
$$

we have the representation (9) on $I_{1} \cup I_{2}$.

By repeating the process mentioned above for the intervals $I_{\kappa}\left(\kappa=1,2, \cdots, \kappa_{0}\right)$ successively, we obtain the representation (9) on the whole interval $I$.

Remark. The fact stated in the above theorem was used by Y. Sibuya without proof in his paper [3].

\section{REFERENCES}

[1] Hirasawa, Y., On solutions of a homogeneous linear matrix equation with variable components, Kodai Math. J., 6 (1983), 70-79.

[2] HIRASAWA, Y., On the construction of linearly independent vectors with variable components, Kodai Math. J., 7 (1984), 34-55. 
[3] Sibuya, Y., Some global properties of matrices of functions of one variable, Math. Ann. 161 (1965), 66-77.

Department of Mathematics

TOKYo Institute of TeCHNOLOGY 